\title{
Dynamical Mean Field Theory and Electronic Structure Calculations
}

\author{
R. Chitra and G. Kotliar \\ Center for Materials Theory, Department of Physics and Astronomy, Rutgers University, Piscataway, NJ 08854, USA
}

(May 1, 2018)

\begin{abstract}
We formulate the dynamical mean field theory directly in the continuum. For a given definition of the local Green's function, we show the existence of a unique functional, whose stationary point gives the physical local Green's function of the solid. We present the diagrammatic rules to calculate it perturbatively in the interaction. Inspired by the success of dynamical mean field calculations for model Hamiltonian systems, we present approximations to the exact saddle point equations which may be used in the strong coupling regime, by using mappings onto generalized quantum impurity models.
\end{abstract}

\section{INTRODUCTION}

The calculation of the electronic structure of solids starting from first principles has been the subject of intensive investigations in the past. The most widely used method is the density functional theory (DFT), used frequently in the local density approximation (for a recent review see Ref. 1). In principle, this method is good for calculating ground state properties only. Time dependent extensions of density functional theoryl have also been formulated and allow the calculation of density and spin correlation response functions which contain information about excited state properties. This method has been very successful in understanding weakly correlated solids but has serious drawbacks when dealing with strongly correlated systems. A second general formulation of the quantum many bodf problem is the Green's function method as formulated early on by Luttinger and Ward 3 and Baym and Kadanoffli. In this method one sets up fully self consistent equations for the one particle Green's function using a functional i.e., an effective action for the Green's function, which has a well defined diagrammatic interpretation in terms of skeleton graphs. However, when considering a realistic band structure, this method becomes fairly intractable beyond the lowest order or GW approximation 5 , and is inadequate for strongly correlated problems.

The purpose of this paper is to describe the foundation of a third approach, namely, the dynamical mean field method (DMFT) in the continuum, in relation to the two other techniques described above. Our main motivation stems from the success of DMFT in dealing with model Hamiltonians of strongly correlated electrons on a lattice $\Theta$. In fact, there are already several works attempting implementations of dynamical mean field ideas to electronic structure calculations]. This paper is an attempt to provide a theoretical underpinning to these computational efforts.

The local spectral function is a central concept in dynamical mean field theory. The goal of the theory is to compute the one electron addition and removal spectra, and quantities which can be naturally expressed in terms of the local spectra. One can view the local spectral function as providing a frequency resolution of the local density. The hope is that by using a more complicated quantity to formulate the theory, one may be able to formulate flexible approximations, which work well even in strongly correlated situations, where density functional theory in the local approximation is known to fail. Indeed, recent work on model calculations has shown that DMFT plays a very useful role in situations where significant changes in the local spectral function take place. These rearrangements of one particle spectral weight may not be accompanied by significant changes in the electron density, and may not be captured well in approximate implementations of density functional theory. This occurs in metal insulator transitions which are accompanied by very small symmetry changes or, small structural charges. Examples of this kind of transition are the paramagnetic insulator to paramagnetic metal transition in $V_{2} \mathrm{O}_{3}$ and $\mathrm{NiSe}_{1-x} S_{x}$, the $\alpha$ to $\gamma$ transition in cerium, the transitions form the $\beta$ to the $\delta$ phase in plutonium or the more recently observed metal insulator transition in $Y_{1-x} T i 0_{3}$ 目. We also expect the dynamical mean field theory to grovide useful descriptions of phases where local moments are well developed but not ordered such as in $\delta$ plutoniume.

In this paper, we will show that in general DMFT functionals are more cumbersome in their diagrammatic formulation than the Ward Luttinger functional for the full Greens function. Nevertheless, our motivation for presenting this formulation here is that DMFT is very promising in terms of practical implementation in very strongly correlated situations. The reason for that is that it can be formulated in terms of impurity models embedded in a medium whose properties have to be evaluated self consistently 10 . A large number of numerical methods for treating the resulting dynamical mean field equations have been developed in the context of model Hamiltonian calculations 9 and these techniques can and should be extended to more realistic models of solids.

Conceptually, the three techniques are very similar: one defines a functional $\Gamma$ of the quantity of interest $\mathrm{X}$ (X being the density in the DFT case, the local Green's function (to be defined later) for DMFT or the full Green's function in 
the Ward Luttinger technique) such that the minimization of $\Gamma$ selects the physical value of the quantity of interest. In all the techniques, there is an exact functional $\Gamma_{\text {exact }}$ having the required property but its explicit analytic form is very difficult to determine. In all the three cases, approximations to $\Gamma_{\text {exact }}$ are necessary to obtain practical results. We adopt a fairly general formulation to set up a diagrammatic expansion for the exact functional of the local Green's function, whose saddle point gives the physical local Green's function. These are presented in the first part of this paper and some possible approximate implementations are presented in the second part of this paper.

Extending the more standard lattice model calculations, our work provides a foundation for doing ab-initio dynamical mean field theory plus electronic structure calculations in the continuum. The goal of our work is twofold. First we want to establish the exact diagrammatic content of $\Gamma$ of the dynamical mean field formalism for realistic models of solids where electrons move in the periodic potential of a crystal, as a formal framework for performing electronic structure calculations or, in other words, to construct a functional for which a dynamical mean field approximation gives the exact answer to the electronic structure problem. This is the content of Sec. II. This perspective should be contrasted with an alternative formulation 11 which will be presented in a separate publication, where dynamical mean field theory will be viewed as an approximate solution to the electronic structure calculation problem. Secondly, in Sec. III, encouraged by the success of dynamical mean field theory in the treatment of model hamiltonians $\mathbf{6}$, we suggest some useful approximations for this functional. This work should provide a conceptual framework for the recent efforts in combining realistic density functional calculations with techniques for solving impurity models in a self consistent environment . Finally, we notice that our work can be regarded as a generalization of Ref. 12, where lattice DMFT was given a functional formulation in terms of a field which is conjugate to the local spectral function. This formulation has given useful insights into the physics of the Mott transition.

\section{EFFECTIVE ACTION FORMALISM}

In this section, we develop the effective action formalism for DMFT. This is in the same spirit as the effective action approach to Kohn-Sham density functional theory (where the bilinear operator is just the electron density) which was developed in a series of publications by Fukuda it et alles and Valiev and Fernando 44 . We first set the notation. The continuum is divided into unit cells labeled by $\mathbf{R}$ and $\mathbf{r}$ is the vector defined within a unit cell. A general "local" bilinear operator has the form

$$
O^{\alpha \beta}\left(\mathbf{r}, \mathbf{r}^{\prime}, \tau, \tau^{\prime}\right)=\int d \mathbf{x} d \mathbf{y} K\left(\mathbf{r}, \mathbf{r}^{\prime} \mid \mathbf{x y}\right) \psi_{\alpha}^{+}(\mathbf{x}, \tau) \psi_{\beta}\left(\mathbf{y}, \tau^{\prime}\right)
$$

where $\psi^{+}$and $\psi$ are the electron creation and annihilation operators and $K$ is a kernel to be specified below. $\mathbf{x}, \tau$ are the space and imaginary time coordinates and $\alpha, \beta$ denote the spin indices. With the definition $\left\langle T_{\tau} O\right\rangle=A\left(\mathbf{r}, \mathbf{r}^{\prime}, \tau, \tau^{\prime}\right)$ (1) leads to

$$
A^{\alpha \beta}\left(\mathbf{r}, \mathbf{r}^{\prime}, \tau, \tau^{\prime}\right)=\int d \mathbf{x} d \mathbf{y} K\left(\mathbf{r}, \mathbf{r}^{\prime} \mid \mathbf{x y}\right) G^{\alpha \beta}\left(\mathbf{x}, \mathbf{y}, \tau, \tau^{\prime}\right)
$$

In terms of the cell indices and unit vectors the space coordinates read $\mathbf{x}=\mathbf{R}+\mathbf{u}$ and $\mathbf{y}=\mathbf{R}^{\prime}+\mathbf{u}^{\prime}$. Here, $\mathbf{u}, \mathbf{r}^{\prime}, \mathbf{r} \mathbf{u}^{\prime}$ are vectors inside a unit cell. The kernel $K$ projects the full $G$ onto a "local" Green's function $A$ defined within a unit cell. By choosing the kernel $K$ to be sufficiently localized around $\mathbf{R}-\mathbf{R}^{\prime} \approx 0$, we extract or project out local information from the full Greens function. There is a great deal of flexibility in deciding what one would like to define as the "local" single particle Green's functions. The simplest choice would be

$$
K=\delta_{\mathbf{R}, \mathbf{R}^{\prime}} \delta\left(\mathbf{r}-\mathbf{r}^{\prime}\right) \delta(\mathbf{r}-\mathbf{u}) \delta\left(\mathbf{r}^{\prime}-\mathbf{u}^{\prime}\right)
$$

which gives a resolution of the density with frequency

$$
\rho(r)=\frac{1}{\beta} \sum_{n} \sum_{\alpha} \exp ^{i \omega_{n} 0^{+}} \bar{A}^{\alpha \alpha}\left(i \omega_{n}, r, r\right)
$$

where $\bar{A}\left(i \omega_{n}, r, r\right)$ is the spectral function corresponding to $\mathrm{A}, \omega_{n}$ are the Matsubara frequencies and $\beta$ is the inverse temperature. Experience has suggested however, that it may be useful to retain local phase information among different orbitals in the local Greens functions. The definition

$$
K=\delta_{\mathbf{R}, \mathbf{R}^{\prime}} \delta(\mathbf{r}-\mathbf{u}) \delta\left(\mathbf{r}^{\prime}-\mathbf{u}^{\prime}\right)
$$


gives us simply the restriction of the full Green's function to a given unit cell. For $\mathbf{r}=\mathbf{r}^{\prime}$, we regain the former choice of local spectral function. Note that (5) and hence the corresponding Green's function $A$ is not invariant under simultaneous lattice translations of $\mathbf{r}$ and $\mathbf{r}^{\prime}$ now viewed as coordinates defined throughout the whole crystal. A kernel with translation invariance is obtained by considering

$$
A^{\alpha \beta}\left(\mathbf{r}, \mathbf{r}^{\prime}, \tau, \tau^{\prime}\right)=\sum_{\mathbf{G}, \mathbf{G}^{\prime}} \sum_{\mathbf{k}} G^{\alpha \beta}\left(\mathbf{k}+\mathbf{G}, \mathbf{k}+\mathbf{G}^{\prime}, \tau, \tau^{\prime}\right) \exp i \mathbf{G} \cdot \mathbf{r}-i \mathbf{G}^{\prime} \cdot \mathbf{r}^{\prime}
$$

Here the $\mathbf{G}$ are the reciprocal lattice vectors and $\mathbf{k}$ runs over the Brillouin zone. Rewriting the right hand side of (6) in real space coordinates and summing over the reciprocal lattice vectors, we obtain a kernel invariant under lattice translations.

$$
K=\sum_{\mathbf{k}} \exp i \mathbf{k} \cdot(\mathbf{x}-\mathbf{y}) \delta(\mathbf{r}-\mathbf{u}) \delta\left(\mathbf{r}^{\prime}-\mathbf{u}^{\prime}\right)
$$

where $\mathbf{k}$ runs over the Brillouin zone. Finally, given a set of tight binding orbitals, there is a natural kernel $K$, associated with this set. The full Green's function is expressed in a set of tight binding orbitals $\phi_{l}$ as follows:

$$
G^{\alpha \beta}(\mathbf{x}, \mathbf{y})=\sum_{l m \mathbf{R}_{1} \mathbf{R}_{2}} \phi_{l}\left(\mathbf{x}-\mathbf{R}_{1}\right) \phi_{m}\left(\mathbf{y}-\mathbf{R}_{2}\right) G_{l m}^{\alpha \beta}\left(\mathbf{R}_{1}, \mathbf{R}_{2}\right)
$$

Similarly, the local Green's function can be projected onto this set as

$$
A^{\alpha \beta}\left(\mathbf{r}, \mathbf{r}^{\prime}\right)=\sum_{l m \mathbf{R}_{1}} \phi_{l}\left(\mathbf{r}-\mathbf{R}_{1}\right) \phi_{m}\left(\mathbf{r}^{\prime}-\mathbf{R}_{1}\right) G_{l m}^{\alpha \beta}\left(\mathbf{R}_{1}, \mathbf{R}_{1}\right)
$$

Using (8), (9) can be rewritten as

$$
A^{\alpha \beta}\left(\mathbf{r}, \mathbf{r}^{\prime}\right)=\sum_{l m \mathbf{R}_{1} \mathbf{R}_{2}} \int_{\mathbf{x}, \mathbf{y}} \phi_{l}\left(\mathbf{x}-\mathbf{R}_{1}\right) \phi_{l}\left(\mathbf{r}-\mathbf{R}_{1}\right) \phi_{m}\left(\mathbf{y}-\mathbf{R}_{2}\right) \phi_{m}\left(\mathbf{r}^{\prime}-\mathbf{R}_{2}\right) G_{l m}^{\alpha \beta}(\mathbf{x}, \mathbf{y})
$$

This leads to the following orbital decomposition for the kernel

$$
K\left(\mathbf{r}, \mathbf{r}^{\prime} \mid \mathbf{x y}\right)=\sum_{l m \mathbf{R}_{1} \mathbf{R}_{2}} \phi_{l}\left(\mathbf{x}-\mathbf{R}_{1}\right) \phi_{l}\left(\mathbf{r}-\mathbf{R}_{1}\right) \phi_{m}\left(\mathbf{y}-\mathbf{R}_{2}\right) \phi_{m}\left(\mathbf{r}^{\prime}-\mathbf{R}_{2}\right)
$$

From these we see that different choices of the basis functions lead to different kernels corresponding to different definitions of the "local" Green's function. We mention that recent workst incorporating DMFT concepts in electronic structure calculations have all used the LMTO15 basis set.

We now use the formalism developed in 13 to construct the effective action $\Gamma$ for the local Green's function defined by (2). These methods prescribe a scheme to cgnstruct an analog of the "Kohn-Sham" correlation potential calculated in the conventional density functional theory 14 . The idea is to construct a perturbative expansion in the interaction strength, for the effective action $\Gamma$. We use the imaginary time formalism in the rest of the paper. The partition function $Z$ for a system of interacting electrons coupled to a source field $J$ via the operator $O$ is

$$
\begin{aligned}
Z\left[J^{\alpha \beta}\right] & =\exp W\left[J^{\alpha \beta}\right] \\
& =\int D \psi D \psi^{+} \exp \left[-S+\int J^{\alpha \beta}\left(\mathbf{r}, \mathbf{r}^{\prime}, \tau, \tau^{\prime}\right) O^{\alpha \beta}\left(\mathbf{r}, \mathbf{r}^{\prime}, \tau, \tau^{\prime}\right)\right]
\end{aligned}
$$

The coordinates $\mathbf{x}$ run over the entire range of the system and It is crucial to note that the indices $\alpha \beta$ are not summed over in the second term in the exponential in (12). The quantity $W[J]$ introduced above is the generator of all connected diagrams. $S$ is the action of a system of electrons with Coulomb interactions between them.

$$
\begin{aligned}
S & =\int d x \sum_{\alpha} \psi_{\alpha}^{+}(x)\left[\partial_{\tau}-\frac{\nabla^{2}}{2 m}+V_{X}(x)\right] \psi_{\alpha}(x) \\
& +\frac{e^{2}}{2} \sum_{\alpha \beta} \int d x d x^{\prime} \psi_{\alpha}^{+}(x) \psi_{\beta}^{+}\left(x^{\prime}\right) U_{c}\left(x-x^{\prime}\right) \psi_{\beta}\left(x^{\prime}\right) \psi_{\alpha}(x)
\end{aligned}
$$


In (14), $x=(\mathbf{x}, \tau), V_{X}$ represents the ionic potential the electron moves in and the Coulomb interaction is $U_{c}\left(x-x^{\prime}\right)=$ $\left|\mathbf{x}-\mathbf{x}^{\prime}\right|^{-1} \delta\left(\tau-\tau^{\prime}\right)$. The effective action $\Gamma$ is defined by the Legendre transform

$$
\Gamma\left[A^{\alpha \beta}\right]=W\left[J_{\alpha \beta}\right]-\int_{\mathbf{r}, \mathbf{r}^{\prime}} J^{\alpha \beta}\left(r, r^{\prime}\right) A^{\alpha \beta}\left(r, r^{\prime}\right)
$$

A naive expectation might be that since $A$ is just a "local" Green's function, the effective action functional $\Gamma$ is given by the usual Baym-Kadanoff functional. Here we find that this is not the case, rather the restriction of $\mathbf{r}, \mathbf{r}^{\prime}$ to a unit cell induces non-trivial corrections to the $\Gamma$ as will be illustrated below. To obtain $\Gamma$ as a functional of $A$, the $J$ dependent terms in (15) should be rewritten in terms of $A$. Since this is not tractable in the presence of interactions, we use the inversion method 13 which involves an expansion of $J, W$ in terms of the coupling constant of the interaction (for example, $e^{2}$ for coulomb interactions) and $J_{0}$, where $J_{0}$ is the source in the absence of the interaction. The crucial ingredient of this method is that $J_{0}$ is chosen so as to reproduce the correct local Green's function $A^{\alpha \beta}$ of the full interacting problem i.e.,

$$
\frac{\delta W_{0}\left[J_{0}^{\alpha \beta}\right]}{\delta J_{0}^{\alpha \beta}}=A^{\alpha \beta}
$$

where $W_{0}$ is the non-interacting free energy. Using (16), the series expansion for the $W\left[J_{0}^{\alpha \beta}\right]$ and $J\left[J_{0}^{\alpha \beta}\right]$ can and rewritten in terms of $A$ and thereby resulting in a series for the effective action $\Gamma\left[A^{\alpha \beta}\right]$

$$
\Gamma\left[A^{\alpha \beta}\right]=\sum_{i=0}^{\infty} e^{2 i} \Gamma_{i}\left[A^{\alpha \beta}\right]
$$

The exact local function $A$ of the interacting system is obtained by minimizing (17)

$$
\frac{\delta \Gamma\left[A^{\alpha \beta}\right]}{\delta A^{\alpha \beta}}=0
$$

\section{A. Diagrammatic rules for $\Gamma$}

The first step is the construction of the non-interacting "Kohn Sham" Green's function $G_{0}\left[J_{0}\right]$

$$
G_{0}^{\alpha \beta}\left(R+r, R^{\prime}+r^{\prime}\right)=\left[\left(\partial_{\tau}-\frac{\nabla^{2}}{2 m}+v_{X}(\mathbf{R}+\mathbf{r})\right) \delta_{\mathbf{R R}^{\prime}} \delta\left(r-r^{\prime}\right) \delta_{\alpha \beta}-J_{0}^{\alpha \beta}\left(r, r^{\prime}\right) \delta_{\mathbf{R}, \mathbf{R}^{\prime}}\right]^{-1}
$$

Note that in the above expression $R+r \equiv\left(\mathbf{R}+\mathbf{r}, \tau_{r}\right)$ and equivalently, $r=\mathbf{r}, \tau_{r} . J_{0}^{\alpha \beta}$ is the Kohn-Sham hybridization function defined earlier. $J_{0}$ is chosen such that $G_{0}$ gives the prescribed local Greens function $A$ of the full interacting problem. Using $G_{0}$, the ferlomb interaction lines $e^{2} U_{c}\left(R-R^{\prime}+r-r^{\prime}\right)$ and a propagator $\mathbf{D}$ to be defined below and following the method of 13.14 , we can obtain $\Gamma$ to all orders in the coupling $e^{2}$. The zeroth order term is $W_{0}=\operatorname{Tr} \log G_{0}$ and the first order term is given by the sum of the Hartree and Fock diagrams shown in Fig.1. The higher order terms which form the Kohn-Sham exchange-correlation potential in the conventional density functional theory are constructed according to the following diagrammatic rules. At every order :

1. Draw all connected diagrams made of Kohn-Sham propagators $G_{0}$ and Coulomb interaction lines $e^{2} U_{c}\left(R-R^{\prime}+r-r^{\prime}\right)$ with the corresponding weight factors.

2. Eliminate all graphs which are one vertex irreducible i.e., separable by cutting a single Coulomb interaction line. 3. For each two-particle reducible (2PR) graph (i.e. any graph that can be separated by cutting two propagator lines), perform the following operations. ${ }^{5}$

(a) Separate the graph by cutting the $2 \mathrm{PR}$ propagators.

(b) For each of the two resulting graphs join two external propagators.

(c) Connect the two graphs via the inverse two particle propagator $\mathbf{D}$ which is the inverse of the connected correlation function $\Pi$ 


$$
\begin{aligned}
\Pi_{\mu \nu \delta \eta}\left(r, r^{\prime}, u, u^{\prime}\right) & =\sum_{\mathbf{R}, \mathbf{R}^{\prime}, \mathbf{U}, \mathbf{U}^{\prime}} \delta_{\mathbf{R}, \mathbf{R}^{\prime}} \delta_{\mathbf{U}, \mathbf{U}^{\prime}}\left\langle\psi_{\mu}^{+}(R+r) \psi_{\nu}\left(R^{\prime}+r^{\prime}\right) \psi_{\delta}^{+}(U+u) \psi_{\eta}\left(U^{\prime}+u^{\prime}\right)\right\rangle \\
& =-\sum_{\mathbf{R}, \mathbf{U}} G_{0}^{\mu \eta}\left(R+r, U+u^{\prime}\right) G_{0}^{\delta \nu}\left(R+r^{\prime}, U+u\right)
\end{aligned}
$$

In the above expressions $U+u \equiv\left(\mathbf{U}+\mathbf{u}, \tau_{u}\right)$. The inverse propagator $\mathbf{D}$ appears in the diagrammatics of the inversion method, as a result of corrections to $J_{0}$ from the interaction which are constrained so that one reproduces the desired local Green's function $A$ order by order in perturbation theory.

(d) Repeat the procedure until no new graph is produced.

(e) Sum up all the resulting graphs including the original graph.

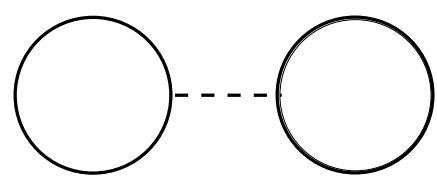

(a)

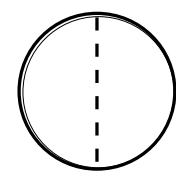

(b)

FIG. 1. a) Hartree and b) Fock diagrams

In Fig.2, we show the second order diagrams which contribute to $\Gamma$. Here the full lines denote particle propagators $G_{0}$ and the dashed line is the Coulomb vertex. Figs. 2a and $2 \mathrm{~b}$ are examples of diagrams which are both one vertex and 2 particle irreducible.

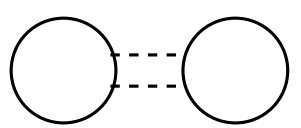

(a)

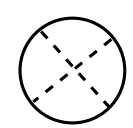

(b)

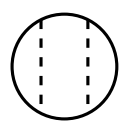

(c)

FIG. 2. Diagrams at second order

However, since the diagram in Fig. 2c is two particle reducible, it transforms according to Rule 3 as shown in Fig. 3. The thick double line denotes the two particle inverse propagator $\mathbf{D}$.

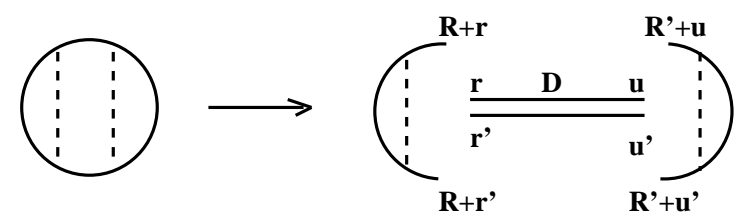

FIG. 3. Dressing of a two particle reducible diagram

\section{B. Self-consistency}

The self-consistent procedure for obtaining the full $A$ is of the same kind as that prescribed for $\Gamma[\rho]$ in Ref. 14 .

- Choose a $J_{0}^{\alpha \beta}\left(r, r^{\prime}\right)$.

- Substitute this in (19) to obtain the Green's function $G_{0}^{\alpha \beta}\left(R+r, R^{\prime}+r^{\prime}\right)$.

- Use this $G_{0}^{\alpha \beta}$ to evaluate the series for $\Gamma \equiv \Gamma_{0}+\Gamma_{H}+\Gamma_{x c}$. Here $\Gamma_{H}$ denotes the Hartree contribution and $\Gamma_{x c}$ represents the contribution of the Fock and all higher order diagrams to $\Gamma$. Determine the new "Kohn-Sham" $J_{0}^{\alpha \beta}$ defined as

$$
J_{0}^{\alpha \beta}=-\left[\frac{\delta \Gamma_{H}}{\delta A^{\alpha \beta}}+\frac{\delta \Gamma_{x c}}{\delta A^{\alpha \beta}}\right]
$$


- Using the $J_{0}$ determined by $(21)$, go back to step 1 and iterate until self-consistency is achieved.

In density functional theory, the second term in (21) is called the exchange-correlation potential $V_{x c}$. The scheme presented above, hinges on the fact that the Legendre transform defined in (15) is invertible. Mathematically, neglecting the spin indices, the transformation is invertible if $\delta^{2} \Gamma / \delta A^{2}$ has no zero eigenvalues. Within the inversion method where $\Gamma$ is evaluated perturbatively in the interaction strength, the invertibility condition described above reduces to the following equation :

$$
\left(\frac{\delta W_{0}\left[J_{0}\right]}{\delta J_{0}}-A\right) \frac{\delta J_{0}}{\delta A}=0
$$

This yields (16) provided $\delta J_{0} / \delta A$ has no zero eigenvalues or equivalently,

$$
\frac{\delta^{2} \Gamma_{0}}{\delta A^{2}} \neq 0
$$

Typically these transformations are invertible for generic values of the variable $A$. Non invertibility is related to a bifurcation of the stationary solution of the functional $\Gamma$ and signals a phase transition. This was exploited in Ref. 12, to analyze the energetics of a system near a Mott transition. The functional constructed here can be used in conjunction with the techniques of Ref. 12, to provide more realistic descriptions of electronic phase transitions. Finally, we mention that we have not yet addressed rigorously, the questions corresponding to the issues of $v$ and $N$ representabilities in density functional theory i.e., what is the domain of definition of our functional or equivalently, what is the range of the function $A$ ?. The formal construction presented here is valid for $A$ 's which are Kohn Sham representable, and we know that the physical Green's function is Kohn Sham representable, with $J_{0}$ being the physical "local" self energy.

\section{Example}

For the sake of clarity, we consider the case of spinless electrons with non-local interactions. In this case,

$$
\Pi\left(r, r^{\prime}, u, u^{\prime}\right)=-\sum_{\mathbf{R}, \mathbf{U}} G_{0}\left(R+r, U+u^{\prime}\right) G_{0}\left(R+r^{\prime}, U+u\right)
$$

We present the various terms upto second order in $e^{2}$, contributing to $\Gamma[A]$ for the kernel of (5). The zeroth order term is $\Gamma_{0}[A]=\operatorname{Tr} \log G_{0}-J_{0} A$. At first order, we have the contributions from the Hartree and Fock diagrams shown in Figs. 1a and 1b.

$$
\Gamma_{1}^{H}=\int d r d r^{\prime} \sum_{\mathbf{R}, \mathbf{R}^{\prime}} G_{0}(R+r, R+r) G_{0}\left(R^{\prime}+r^{\prime}, R^{\prime}+r^{\prime}\right) U_{c}\left(R-R^{\prime}+r-r^{\prime}\right)
$$

The contribution from the Fock term is as follows:

$$
\Gamma_{1}^{F}=\int d r d r^{\prime} \sum_{\mathbf{R}, \mathbf{R}^{\prime}} G_{0}\left(R+r, R^{\prime}+r^{\prime}\right) G_{0}\left(R^{\prime}+r^{\prime}, R+r\right) U_{c}\left(R-R^{\prime}+r-r^{\prime}\right)
$$

Similarly the diagrams which contribute to second order are shown in Figs.(2a-2c).

$$
\begin{aligned}
\Gamma_{2}^{a}= & \int d r d r^{\prime} d u d u^{\prime} \sum_{\mathbf{R}, \mathbf{R}^{\prime} \mathbf{R}_{1}, \mathbf{R}_{1}^{\prime}} G_{0}\left(R+r, R_{1}+u\right) G_{0}\left(R_{1}+u, R+r\right) \\
& U_{c}\left(R-R^{\prime}+r-r^{\prime}\right) G_{0}\left(R^{\prime}+r^{\prime}, R_{1}^{\prime}+u^{\prime}\right) U_{c}\left(R_{1}-R_{1}^{\prime}+u-u^{\prime}\right) G_{0}\left(R_{1}^{\prime}+u^{\prime}, R^{\prime}+r^{\prime}\right) \\
\Gamma_{2}^{b}= & \int d r d r^{\prime} d u d u^{\prime} \sum_{\mathbf{R}, \mathbf{R}^{\prime} \mathbf{R}_{1}, \mathbf{R}_{1}^{\prime}} G_{0}\left(R+r, R_{1}+u\right) G_{0}\left(R_{1}+u, R^{\prime}+r^{\prime}\right) U_{c}\left(R-R^{\prime}+r-r^{\prime}\right) \\
& G_{0}\left(R^{\prime}+r^{\prime}, R_{1}^{\prime}+u^{\prime}\right) U_{c}\left(R_{1}-R_{1}^{\prime}+u-u^{\prime}\right) G_{0}\left(R_{1}^{\prime}+u^{\prime}, R+r\right)
\end{aligned}
$$




$$
\begin{aligned}
\Gamma_{2}^{c}= & \int d r d r^{\prime} d u d u^{\prime} \sum_{\mathbf{R}, \mathbf{R}^{\prime} \mathbf{R}_{1}, \mathbf{R}_{1}^{\prime}} G_{0}\left(R+r, R_{1}+u\right) G_{0}\left(R_{1}+u, R_{1}^{\prime}+u^{\prime}\right) \\
& U_{c}\left(R-R^{\prime}+r-r^{\prime}\right) G_{0}\left(R_{1}^{\prime}+u^{\prime}, R^{\prime}+r^{\prime}\right) U_{c}\left(R_{1}-R_{1}^{\prime}+u-u^{\prime}\right) G_{0}\left(R^{\prime}+r^{\prime}, R+r\right)
\end{aligned}
$$

Since Fig.2c is $2 \mathrm{PR}$, following the prescription (3a-3c) presented earlier, we subtract the following term from the $2 \mathrm{PR}$ diagram of (31)

$$
\begin{aligned}
\Gamma_{2}^{c r}= & \int \sum_{\mathbf{R}, \mathbf{R}^{\prime}, \mathbf{R}_{1}, \mathbf{R}_{1}^{\prime}, \mathbf{R}_{2}, \mathbf{R}_{2}^{\prime}} G_{0}\left(R_{1}+r_{1}, R_{1}^{\prime}+r_{1}^{\prime}\right) G_{0}\left(R_{1}+r_{1}, R+r\right) G_{0}\left(R_{1}^{\prime}+r_{1}^{\prime}, R+r^{\prime}\right) \Pi^{-1}\left(r, r^{\prime}, u, u^{\prime}\right) \\
& G_{0}\left(R^{\prime}+u, R_{2}+r_{2}\right) G_{0}\left(R_{2}^{\prime}+r_{2}^{\prime}, R^{\prime}+u^{\prime}\right) G_{0}\left(R_{2}+r_{2}, R_{2}^{\prime}+r_{2}^{\prime}\right) U_{c}\left(R_{1}-R_{1}^{\prime}+r_{1}-+r_{1}^{\prime}\right) U_{c}\left(R_{2}-R_{2}^{\prime}+r_{2}-+r_{2}^{\prime}\right)
\end{aligned}
$$

The integrations are over imaginary time and all the vectors within a unit cell. Therefore, the contribution to $\Gamma$ at second order is

$$
\Gamma_{2}=\frac{1}{2}\left(\Gamma_{2}^{b}-\Gamma_{2}^{a}\right)+\left(\Gamma_{2}^{c}-\Gamma_{2}^{c r}\right)
$$

If we consider the case where the coordinates $\mathbf{u}, \mathbf{r}$ scan the entire space i.e., $K=1$ and $A=G(x, y)$, we find that the structure of $\Pi$ yields $\Gamma_{2}^{c r}=\Gamma_{2}^{c}$, hence canceling the contribution of the 2PR diagram of Fig.2c. Since such cancellations occur at all orders, the rules (1-3) imply that only two particle irreducible (2PI) are retained in the expansion for $\Gamma$. In addition, (16) implies that $G_{0}=G$ and $J_{0}=G_{n}^{-1}-G^{-1}$, where $G_{n}$ is the non-interacting Green's function in the absence of the source. Replacing $G_{0}$ by $G$ in the above terms, we recover the Baym Kadanoff functional for the Green's function $G$

$$
\Gamma[G]=\operatorname{Tr} \log G-\operatorname{Tr} G_{n}^{-1} G+\Gamma_{1}+I_{2}[G]
$$

where $I_{2}$ is the sum of all connected two particle irreducible diagrams occurring at second and higher orders.

\section{D. generalization}

The formalism presented above can be used to construct the effective action functional for any arbitrary choice of the operator $O$ or equivalently, the kernel $K$ in (11). The rules (1-3) can be extended directly, provided $K$ satisfies the following conditions:

1. $K\left(\mathbf{r}, \mathbf{r}^{\prime} \mid \mathbf{x}, \mathbf{y}\right)$ is invertible

2. $\int K^{-1}\left(\mathbf{r}, \mathbf{r}^{\prime} \mid \mathbf{x}, \mathbf{y}\right) K\left(\mathbf{r}, \mathbf{r}^{\prime} \mid \mathbf{w}, \mathbf{z}\right) d \mathbf{r} d \mathbf{r}^{\prime}=\delta_{\mathbf{w x}} \delta_{\mathbf{y z}}$

3. $\int K^{-1}\left(\mathbf{r}, \mathbf{r}^{\prime} \mid \mathbf{x}, \mathbf{y}\right) K\left(\mathbf{u}, \mathbf{u}^{\prime} \mid \mathbf{x}, \mathbf{y}\right) d \mathbf{x} d \mathbf{y}=\delta_{\mathbf{r u}} \delta_{\mathbf{r}^{\prime} \mathbf{u}^{\prime}}$

We can then use the rules (1-3) to construct $\Gamma[A]$ with the following replacements

$$
G_{0}\left(R+r, R^{\prime}+r^{\prime}\right)=\left[\left(\partial_{\tau}-\frac{\nabla^{2}}{2 m}+v_{X}(\mathbf{R}+\mathbf{r})\right) \delta_{\mathbf{R R}^{\prime}} \delta\left(r-r^{\prime}\right)+\int d \mathbf{u} d \mathbf{u}^{\prime} J\left(\mathbf{u}, \mathbf{u}^{\prime}, \tau, \tau^{\prime}\right) K\left(\mathbf{u}, \mathbf{u}^{\prime} \mid \mathbf{R}+\mathbf{r}, \mathbf{R}^{\prime}+\mathbf{r}^{\prime}\right)\right]^{-1}
$$

and

$$
\begin{aligned}
\Pi\left(r, r^{\prime}, u, u^{\prime}\right) & =\sum_{\mathbf{R}, \mathbf{R}^{\prime}, \mathbf{U}, \mathbf{U}^{\prime}} \int_{\mathbf{a}, \mathbf{a}^{\prime}, \mathbf{b}, \mathbf{b}^{\prime}} K\left(\mathbf{r}, \mathbf{r}^{\prime} \mid \mathbf{R}+\mathbf{a}, \mathbf{R}^{\prime}+\mathbf{a}^{\prime}\right) K\left(\mathbf{u}, \mathbf{u}^{\prime} \mid \mathbf{U}+\mathbf{b}, \mathbf{U}^{\prime}+\mathbf{b}^{\prime}\right)\left\langle\psi^{+}(R+a) \psi\left(R^{\prime}+a^{\prime}\right) \psi^{+}(U+b) \psi\left(U^{\prime}+b^{\prime}\right)\right\rangle \\
& =\sum_{\mathbf{R}, \mathbf{R}^{\prime}, \mathbf{U}, \mathbf{U}^{\prime}} \int_{\mathbf{a}, \mathbf{a}^{\prime}, \mathbf{b}, \mathbf{b}^{\prime}} K\left(\mathbf{r}, \mathbf{r}^{\prime} \mid \mathbf{R}+\mathbf{a}, \mathbf{R}^{\prime}+\mathbf{a}^{\prime}\right) K\left(\mathbf{u}, \mathbf{u}^{\prime} \mid \mathbf{U}+\mathbf{b}, \mathbf{U}^{\prime}+\mathbf{b}^{\prime}\right) G_{0}\left(R+a, U^{\prime}+b^{\prime}\right) G_{0}\left(R^{\prime}+a^{\prime}, U+b\right) \quad(36)
\end{aligned}
$$

The generalization to the spin dependent case is straightforward. 


\section{APPROXIMATIONS}

We now address the question of how to calculate $\Gamma_{x c}$ in (21). As is the case in the usual density functional theory, the evaluation of all the diagrams that contribute to $\Gamma$ and hence $\Gamma_{x c}$ is impossible and one has to take recourse to various approximation schemes depending on the strength of the interactions.

\section{A. Weak Coupling}

The simplest scheme would be to use truncated perturbation theory to second order, utilizing the expressions given in the previous section. Alternatively, one could use a random phase approximation. This approximation leads to a simplification of the rules for the effective action presented above. Since all RPA diagrams are by definition irreducible with respect to the coulomb vertex and two particle irreducible, we do not have to go through the complicated procedure outlined in rules $(3 \mathrm{a})-(3 \mathrm{e})$ to deal with reducible diagrams. It would be interesting to investigate the equivalent of the local density approximation (LDA) for these new functionals, which corresponds to evaluating the diagrammatic expansion for $\Gamma_{x c}$ described in the previous section for a spectral function which is independent of the spatial coordinates i.e., spatially homogeneous. Unfortunately, the energy of the uniform electron gas expressed as a functional of its spectral function is not available and further work is required before a correspondence with LDA can be made.

\section{B. Strong Coupling - Impurity models}

The weak coupling approximations for $\Gamma$ can be set up for an arbitrary kernel $K$ in (11). On the other hand, different strong coupling approximations can be set up for differept choices of the kernel and are closely related to the formalism of dynamical mean field theory (DMFT) on the lattice 6 , where the local Greens function is represented as a Greens function of a generalized Anderson impurity model. In finite dimensions, the fact that the exact Green's function can be represented in terms of a local impurity model is an assumption. It suggests however, the implementation of the local Green's function defined by the kernel in (5). For simplicity, we consider the paramagnetic case in the following. Denoting the unit cell by B we represent the local Greens function defined by the kernel (5), as a generalized impurity model described by an action

$$
\begin{aligned}
S\left\{G_{o}\right\} & =\int d \tau d \tau^{\prime} \int_{B} d \mathbf{r} \int_{B} d \mathbf{r}^{\prime} \sum_{\alpha} \psi_{\alpha}^{+}(r) A_{o}^{-1}\left(r, r^{\prime}\right) \psi_{\alpha}\left(r^{\prime}\right) \\
& +\int_{o}^{\beta} d \tau d \tau^{\prime} \int_{B} d \mathbf{r} \int_{B} d \mathbf{r}^{\prime} \rho(r) V_{S R}\left(r, r^{\prime}\right) \rho\left(r^{\prime}\right)
\end{aligned}
$$

Here $r \equiv(\mathbf{r}, \tau)$ and the density $\rho(r)=\sum_{\alpha} \psi_{\alpha}^{+}(r) \psi_{\alpha}(r)$. The interaction in the full continuum model is now replaced by an effective short ranged interaction $V_{S R}\left(r, r^{\prime}\right)=V_{S R}\left(\mathbf{r}, \mathbf{r}^{\prime}\right) \delta\left(\tau-\tau^{\prime}\right)$ within the cell $B$. For lattice models, such a mapping led one from the full Green's function $G_{i j}$ to a local Green's function $G_{i i}$. In the continuum, the mapping leads to a similar "local" Green's function $A\left(r, r^{\prime}\right)$ which is the Green's function defined within the unit cell. Referring back to Sec.II, we see that a simple generalization of DMFT ideas automatically leads us to consider (5) as the simplest choice for local Green's function in the continuum. Analogy with DMFT on a lattice, leads to write a self consistent equation for $A_{o}$ by

$$
A_{o}^{-1}\left(r, r^{\prime}\right)=\left[\frac{\partial}{\partial \tau}-\frac{\nabla^{2}}{2 m}-\mu-V_{X}(r)-V_{H}(r)\right] \delta\left(\tau-\tau^{\prime}\right) \delta\left(\mathbf{r}-\mathbf{r}^{\prime}\right)+\Delta\left(r, r^{\prime}\right)
$$

where $V_{H}$ is the Hartree potential that arises naturally in such an impurity mapping and $\Delta\left(r, r^{\prime}\right)$ is the time-dependent "Weiss field of the problem". It describes the effect of all the other unit cells (which have been integrated out), on the selected unit cell. Note that this looks like a generalization of the multi-orbital impurity models if we assume that the orbital indices are the continuous variables $\mathbf{r}, \mathbf{r}^{\prime}$. Using $S\left(A_{o}\right)$ given in (37) we can compute the "local Green's function" $A\left(r, r^{\prime}\right)$ of the unit cell defined by

$$
\frac{\int d \psi^{+} d \psi \psi(r) \psi\left(r^{\prime}\right) e^{S\left(A_{o}\right)}}{\int e^{S\left\{A_{o}\right\}}}=A\left(r, r^{\prime}\right)\{\Delta\}
$$


Note that $A$ depends on the Weiss field $\Delta$. The self energy $\Sigma[\Delta]=A_{o}^{-1}-A^{-1}\left(r, r^{\prime}\right)$ can be thought of as the sum of the Hartree term plus all the two particle irreducible skeleton graphs with interaction lines $V_{S R}\left(r, r^{\prime}\right)$ and particle lines given by the "local" Green's function $A\left(r, r^{\prime}\right)$. Using the above, the self consistency condition for $\Delta\left(r, r^{\prime}\right)$ is obtained by requiring that

$$
A^{-1}\left(r, r^{\prime}\right)[\Delta]=\left[\frac{\partial}{\partial \tau}-\frac{\nabla^{2}}{2 m}-\mu-V_{X}(r)-V_{H}(r)[\Delta]\right] \delta\left(\mathbf{r}-\mathbf{r}^{\prime}\right) \delta\left(\tau-\tau^{\prime}\right)+\Delta\left(r, r^{\prime}\right)-\Sigma\left(r, r^{\prime}\right)[\Delta]
$$

This procedure of mapping the model onto an impurity model with interactions defined only within the unit cell is nothing but an approximation to the infinite series of diagrams in the perturbative expansion described earlier. Diagrammatically, the present method retains only local diagrams i.e., those whose internal propagators and vertices just connect points within the same unit cell. None of the diagrams include particle lines going from one unit cell to another. This is indeed reminiscent of the Hubbard model calculations in infinite dimensions where the self energy is completely local in space. A solution of this self-consistent equation for $\Delta$ is sufficient to specify the local $A$ and hence other dynamical quantities.

The ahoye mapping can be generalized to a more general impurity model which goes under the name of extended DMFT 16.17. This captures some important aspects of the long range part of the Coulomb interaction 17 . In the case of lattice models, one obtains a single impurity whose on-site interaction is dynamically and self-consistently screened due to the presence of long-ranged coulomb interactions in the original lattice model. This has been worked out explicitly for the lattice model in Ref. 17. A straight forward generalization to the continuum is obtained by replacing the short-ranged interaction $V_{S R}\left(r, r^{\prime}\right)$ in (37), by the retarded time dependent interaction

$$
\Pi_{o}^{-1}\left(r, r^{\prime}\right)=V_{S R}\left(\mathbf{r}, \mathbf{r}^{\prime}\right) \delta\left(\tau-\tau^{\prime}\right)+\Omega\left(r, r^{\prime}\right)
$$

In the same manner as above, the local density density correlation function $\Pi=\left\langle\rho(r) \rho\left(r^{\prime}\right)\right\rangle$ is given by

$$
\Pi^{-1}\left(r, r^{\prime}\right)[\Omega, \Delta]=\Pi_{o}^{-1}-\tilde{\Pi}[\Omega, \Delta]
$$

where $\tilde{\Pi}$ is the irreducible part of $\Pi$. We, therefore, see that since $\Pi$ depends on the retarded correlations, the dynamical interaction $\Pi_{o}$ is also determined in a self-consistent manner. Moreover, in the presence of this retarded interaction, the self energy $\Sigma=\Sigma[\Delta, \Omega]$ i.e., it depends on $\Delta$ through $G_{o}$ and also on $\Omega$ through the interaction $\Pi_{o}$. Therefore, (40) and (42) form a set of coupled equations for $\Delta$ and $\Omega$ which have to be solved self-consistently. These are the generalizations of the extended DMFT equations obtained in Ref. 17. The diagrammatic content of this approach is slightly different from that of the unscreened model since the vertex $V_{S R}$ is now replaced by $\Pi_{o}$. This implies that in the full continuum model, we retain all those "local" diagrams with internal particle lines $A\left(r, r^{\prime}\right)$ and long ranged vertices which connect any pair of arbitrary points $\mathbf{r}+\mathbf{R}$ and $\mathbf{R}^{\prime}+\mathbf{r}^{\prime}$.

To summarize, we see that DMFT ideas can be generalized to provide strong coupling approximations to the finite dimensional effective actions of the local Green's function $A$. We briefly mention that the above mappings can be generalized to include the effects of magnetic ordering. In this paper, we have presented the generalized impurity model only for the simplest kernel and it would be interesting to study whether there exist other generalized impurity models for arbitrary choices of the local Green's functions. In particular we notice that current DMFT calculations using realistic multiband Hamiltonians $\mathrm{G}$ based on LMTO orbitals can be viewed as approximate realizations of the functional of the local Green's function based on the choice of the kernel (11), where the tight binding basis defining the kernel are currently obtained from the self consistent solutions of a density functional calculation.

\section{CONCLUSIONS}

In this paper, we have described a means of studying electronic structure problems defined in the continuum, involving effective action formalisms and the principles of dynamical mean field theories. We first outlined the method to formulate the effective action functional for a general "local" Green's function. These local functions are good probes of the dynamics of the system including metal insulator transitions. Though we present a scheme to calculate these functionals to every order in the interaction, as is the case with standard density functional theory, an exact evaluation of these functionals is practically intractable. We have, therefore, described various weak and strong coupling approximations. The strong coupling approximations are a generalization of the DMFT ideas on a lattice to the continuum. Recently, some of these generalized DMFT ideas were applied to the problem of the Hubbard model with coulomb long range interactions.17. We found that the long range interactions screen the effective onsite interaction in such a manner as to make the continuous Mott transition seen in that system, discontinuous. These results suggest that a direct implementation of DMFT to an electronic structure problem can produce results 
which are not just quantitatively but also qualitatively different from those obtained from conventional DMFT model calculations.

${ }^{1}$ For a recent review see N. Argaman and G. Makov, physics/9806013 and references therein.

${ }^{2}$ E.K.U. Gross and W. Kohn, Adv. Quant. Chem. 21, 255 (1990).

3 J.M. Luttinger and J.C. Ward, Phys. Rev. 118, 1417 (1960).

${ }^{4}$ G. Baym and L.P. Kadanoff, Phys. Rev. 124, 287 (1961).

${ }^{5}$ F. Aryasetiawan and O. Gunnarsson Rep. Prog. Phys. 61 (1998) 237

${ }^{6}$ A.Georges, G. Kotliar, W. Krauth and M. Rozenberg, Rev. Mod. Phys. 68, (1996) 13.

${ }^{7}$ V. Anisimov et. al. J. Phys. Cond. Matt 9 (1997) 7359 A Lichtenstein and M. Katsenelson Phys. Rev. B 57, 6884 (1988).

${ }^{8}$ M. Kasuya et. al. Phys. Rev. B 47, 6197 (1993).

${ }^{9}$ S. Savrasov and G. Kotliar cond-mat/9908401.

10 A. Georges and G. Kotliar, Phys. Rev. B 15, 6479-6483 (1992).

${ }^{11}$ R. Chitra and G. Kotliar, to be submitted.

${ }^{12}$ G. Kotliar European Journal of Physics B, 11, 27-39 (1999).

${ }^{13}$ R. Fukuda T. Kotani and S. Yokojima Prog. Theory Phys. 92, 833 (1994); R. Fukuda et. al. Prog. Theory Phys. Suppl. 121, 1 (1996)

${ }^{14}$ M. Valiev and G. Fernando Phys. Lett. A 227, 265 (1997)

${ }^{15}$ O.K. Andersen, Phys. Rev. B 12, 3060 (1975).

${ }^{16}$ Q. Si and J.L. Smith, Phys. Rev. Lett 77, 3391 (1997). H. Kajueter Rutgers University Ph.D. Thesis 1996. H. Kajueter and G. Kotliar Rutgers University preprint (1996);

${ }^{17}$ R. Chitra and G. Kotliar, cond-mat 9903185. 